\title{
Limiting top quark-Higgs boson interaction and Higgs-boson width from multitop productions
}

\author{
Qing-Hong Cao, ${ }^{1,2,3, *}$ Shao-Long Chen, ${ }^{4,3, \dagger}$ Yandong Liu, ${ }^{5,6, *}$ Rui Zhang, ${ }^{1,}$ and Ya Zhang ${ }^{1, \|}$ \\ ${ }^{1}$ Department of Physics and State Key Laboratory of Nuclear Physics and Technology, \\ Peking University, Beijing 100871, China \\ ${ }^{2}$ Collaborative Innovation Center of Quantum Matter, Beijing 100871, China \\ ${ }^{3}$ Center for High Energy Physics, Peking University, Beijing 100871, China \\ ${ }^{4}$ Key Laboratory of Quark and Lepton Physics (MoE) and Institute of Particle Physics, \\ Central China Normal University, Wuhan 430079, China \\ ${ }^{5}$ Key Laboratory of Beam Technology of Ministry of Education, College of Nuclear Science \\ and Technology, Beijing Normal University, Beijing 100875, China \\ ${ }^{6}$ Beijing Radiation Center, Beijing 100875, China
}

(Received 19 January 2019; published 25 June 2019)

\begin{abstract}
We demonstrate that the multitop productions efficiently probe the $C P$ property of a top quark-Higgs boson interaction and the Higgs boson width at the LHC. The four top-quark production alone can exclude a purely $C P$-odd top-quark Yukawa coupling at the $13 \mathrm{TeV}$ LHC with an integrated luminosity of $680 \mathrm{fb}^{-1}$, regardless the size of the Yukawa coupling. Combining the single Higgs-boson production, the $t \bar{t} H$ associated production, and the four top-quark production, we show that the $C P$ phase of the top-quark Yukawa coupling and the Higgs-boson width can be stringently bounded at the LHC with integrated luminosities of $300 \mathrm{fb}^{-1}$ and $3000 \mathrm{fb}^{-1}$.
\end{abstract}

DOI: $10.1103 /$ PhysRevD.99.113003

\section{INTRODUCTION}

Measurement of the rate of the association production of a top-quark pair and a Higgs boson (named as the $t \bar{t} H$ channel) provides a direct test of the Higgs-boson interaction with the top-quark, i.e., the so-called top-quark Yukawa interaction. Recently, both the ATLAS and CMS Collaborations confirm the top-quark Yukawa coupling $\left(y_{t}\right)$ at the $5 \sigma$ C.L. [1-3]. The next task is to test the $C P$ property of $y_{t}$ [4-6]. As the strength of $y_{t}$ is comparable to the coupling strength of strong interaction $\left(g_{s}\right)$, it has been shown that the four top-quark $(t \bar{t} t \bar{t})$ production can probe the $C P$-even $y_{t}$ coupling and also the tiny Higgs-boson width at the LHC and future colliders [7-10]. In this work, we first illustrate that the $t \bar{t} t \bar{t}$ production is good at probing the $C P$ property of the $y_{t}$ coupling, and then perform a global fit to explore the sensitivity of the $13 \mathrm{TeV}$ LHC on $y_{t}$

\footnotetext{
*qinghongcao@pku.edu.cn

chensl@mail.ccnu.edu.cn

¥ydliu@bnu.edu.cn

zhang.rui@pku.edu.cn

zhangya1221@pku.edu.cn
}

Published by the American Physical Society under the terms of the Creative Commons Attribution 4.0 International license. Further distribution of this work must maintain attribution to the author(s) and the published article's title, journal citation, and DOI. Funded by SCOAP ${ }^{3}$. after combining the single-Higgs production and $t \bar{t} H$ production.

The Higgs-boson width plays a crucial role in all the Higgs-boson productions. Even though in the Standard Model (SM) it is very tiny $(\sim 4 \mathrm{MeV})$ and hard to be measured, the Higgs-boson width could distinguish various new physics models, e.g., the composite Higgs model or Higgs-portal dark matter models including Higgs invisible decays, etc. As the Higgs width is much less than the detector energy resolution, $\sim 1 \mathrm{GeV}$, one can extract it through the comparison of the processes involving the on shell and off shell Higgs boson. One way is to examine the on shell and off shell Higgs-boson effect in the $g g \rightarrow$ $H \rightarrow Z Z^{*}$ process [11]. Another way is to compare the $t \bar{t} H$ and $t \bar{t} t \bar{t}$ productions [7]. In this study, we explore the sensitivity of the LHC to the Higgs-boson width through the multitop productions.

The general top-quark Yukawa coupling $y_{t}$ is parametrized as following:

$$
\mathcal{L}_{t \bar{t} H}=-\frac{m_{t}}{v} H \bar{t}\left(a_{t}+i b_{t} \gamma_{5}\right) t
$$

where $m_{t}$ is the top-quark mass, $v(=174 \mathrm{GeV})$ the vacuum expectation value, and the coefficient $a_{t}\left(b_{t}\right)$ denotes the $C P$-even $(C P$-odd $)$ coupling, respectively. Figure 1 displays the representative Feynman diagrams of the $t \bar{t} t \bar{t}$ production, which occurs either through the gluon 

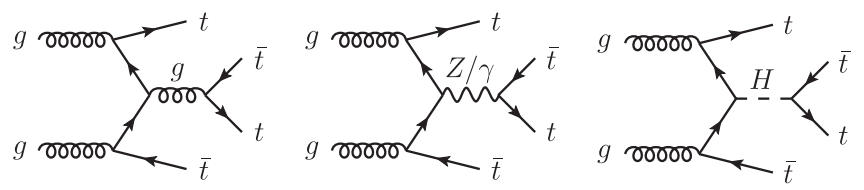

FIG. 1. Illustrative Feynman diagrams of $t \bar{t} t \bar{t}$ productions.

mediation, the electroweak gauge-boson mediation, or the Higgs boson mediation in the SM. We name the corresponding matrix elements as $\mathcal{M}_{g}, \mathcal{M}_{Z / \gamma}$, and $\mathcal{M}_{H}$. The contribution of $\mathcal{M}_{H}$ to the $t \bar{t} t \bar{t}$ production cross section is proportional to $y_{t}^{4}$, and that enables us to measure $y_{t}$ through the $t \bar{t} t \bar{t}$ channel. We assume QCD and electroweak interactions of top quark are the same as other light quarks and do not consider the uncertainties of QCD and electroweak interactions of the top quark. See Ref. [12] for the global fitting result of top quark QCD and electroweak couplings. In the study, we consider only the top Yukawa coupling might differ from the SM value. The cross section of $t \bar{t} t \bar{t}$ production is parametrized as follows:

$$
\sigma(t \bar{t} t \bar{t})=\sigma(t \bar{t} t \bar{t})_{g+Z / \gamma}+\sigma(\bar{t} \bar{t} t \bar{t})_{\mathrm{int}}+\sigma(t \bar{t} t \bar{t})_{H},
$$

where

$$
\begin{aligned}
\sigma(t \bar{t} t \bar{t})_{g+Z / \gamma} & \propto\left|\mathcal{M}_{g}+\mathcal{M}_{Z / \gamma}\right|^{2}, \\
\sigma(t \bar{t} t \bar{t})_{\mathrm{int}} & \propto \mathcal{M}_{g+Z / \gamma} \mathcal{M}_{H}^{\dagger}+\mathcal{M}_{g+Z / \gamma}^{\dagger} \mathcal{M}_{H}, \\
\sigma(t \bar{t} t \bar{t})_{H} & \propto\left|\mathcal{M}_{H}\right|^{2} .
\end{aligned}
$$

We calculate $\sigma(t \bar{t} t \bar{t})$ at the leading order using MadEvent [13] in terms of the $H_{T} / 4$ scale scheme and PDFsets of LUXqed_plus_PDF4LHC15_nnlo_100, which yields

$$
\begin{aligned}
\sigma(t \bar{t} t \bar{t})_{13 \mathrm{TeV}}= & 7.724-1.164 a_{t}^{2}+2.434 b_{t}^{2} \\
& +0.910 a_{t}^{4}+2.183 a_{t}^{2} b_{t}^{2}+1.424 b_{t}^{4},
\end{aligned}
$$

where we ignore mixing terms of $a_{t} b_{t}, a_{t}^{3} b_{t}$, and $a_{t} b_{t}^{3}$ as their contributions are about one thousandth of those terms shown above. The first term on the right-handed side denotes $\sigma(t \bar{t} t \bar{t})_{g+Z / \gamma}$, the second and third terms represent $\sigma(t \bar{t} t \bar{t})_{\text {int }}$, and the last three terms label $\sigma(t \bar{t} t \bar{t})_{H}$.

It is worth noting that, in the interference contribution $\sigma^{\mathrm{SM}}(t \bar{t} t \bar{t})_{\text {int }}$, the $C P$-even contribution $\left(\propto a_{t}^{2}\right)$ is negative while the $C P$-odd contribution $\left(\propto b_{t}^{2}\right)$ is positive. In the $\sigma^{\mathrm{SM}}(t \bar{t} t \bar{t})_{H}$, the $C P$-even and $C P$-odd contributions as well as their interference are all positive. Moreover, the $C P$-odd contribution is larger than that of the $C P$ even in the $\sigma^{\mathrm{SM}}(t \bar{t} t \bar{t})_{\text {int }}$ and $\sigma^{\mathrm{SM}}(t \bar{t} t \bar{t})_{H}$; therefore, it is more promising to observe the pseudoscalar effect through the $t \bar{t} t \bar{t}$ production.

The CMS Collaboration has searched the four top-quark production at the $13 \mathrm{TeV}$ LHC with an integrated luminosity of $35.9 \mathrm{fb}^{-1}$ [8]. The signal significance is $1.6 \sigma$ for the SM process contribution ( $a_{t}=1$ and $b_{t}=0$ ), even after utilizing the combined multiple signal region analysis. It can achieve $2.0 \sigma$ with the data sample collected before the LHC shutdown by means of naive integrated luminosity rescaling. In our study, we adopt a signal region in the collider simulation and analysis. Thus, a larger data sample is needed to exclude/discover the four top-quark production at the $13 \mathrm{TeV}$ LHC; i.e., the integrated luminosity has to be accumulated to $\sim 240 \mathrm{fb}^{-1}$. While for the purely $C P$-odd top quark-Higgs boson interaction $\left(a_{t}=0\right.$ and $\left.b_{t}=1\right)$, it is shown that the integrated luminosity of $\sim 115 \mathrm{fb}^{-1}$ can probe the four top-quark production with a significance of $2 \sigma$ due to the positive interference contribution as shown above. As to be discussed below, the discovery potential further suffers from the renormalization/factorization scale uncertainties; that makes the four-top quark observation more challenging. Taking the scale uncertainty into account, one is able to exclude or discover the purely $C P$-odd top quark-Higgs boson interaction by the four top-quark production at the confidence level of $2 \sigma$ with the integrate luminosity of $\sim 345 \mathrm{fb}^{-1}$. Thanks to the high luminosity of HL-LHC, it is easy to observe the four top quark production, regardless of the scale dependence of the production rate.

In the following study, we separate our analysis into two steps based on the time line of the LHC operation: one is the low luminosity case in which the four-top-quark production is not observed yet; the other is the high luminosity phase in which the four top-quark production is well observed and measured precisely. In the former case, we obtain a bound on the top quark-Higgs boson interaction from the null four top-quark events, while in the latter, we derive the range of the top quark-Higgs boson coupling that produces the observed four-top events.

The complete next-to-leading order (NLO) corrections to the signal process are large and yield a $k$ factor $\left(K_{F}\right)$ as 1.58 for the SM $\left(a_{t}=1\right.$ and $\left.b_{t}=0\right)$ [9]. With the assumption that the $C P$ property of the top quark-Higgs boson interaction affects the NLO corrections mildly, in the study, we rescale the $\mathrm{LO}$ cross section of the signal process by an overall $K_{F}=1.58$ to mimic the high order QCD effects in both the $C P$-even and $C P$-odd cases; i.e., we multiply a global $k$ factor in Eq. (4). The drawback of the $t \bar{t} t \bar{t}$ production is that it suffers from a large dependence on the choices of the renormalization scale and the factorization scale $[14,15]$. Even worse, including higher order quantum corrections only improves the scale dependence mildly [9]. We conservatively consider the cross section of the signal process exhibits a $50 \%$ uncertainty of the scale dependence throughout our study, i.e.,

$$
\sigma=\sigma_{0} \pm \delta \sigma_{\mu}, \quad \delta \sigma_{\mu}=50 \% \times \sigma_{0},
$$

where $\sigma_{0}$ denotes the central value of the cross section and $\delta \sigma_{\mu}$ is the variation from the scale dependence. 


\section{PURELY CP-ODD $y_{t}$ COUPLING}

In the previous study [7], we explored the potential of the LHC on the $C P$-even $y_{t}$, considering the event topology of the $t \bar{t} t \bar{t}$ production consists of same-sign charged leptons, lots of (b) jets and missing transverse momentum. Such a collider signature has been studied by the CMS Collaboration [8] to constrain a purely $C P$-even $y_{t}$. Now, we first extend our study to the purely $C P$-odd $y_{t}$ and then address the $C P$-mixture case. To probe purely $C P$-odd top quark-Higgs boson coupling, we consider two benchmark cases: $C P$-odd I $\left(a_{t}=0\right.$ and $\left.b_{t}=1\right)$ and $C P$-odd II $\left(a_{t}=0\right.$ and $b_{t}$ free).

The kinematics distributions of the final state particles are affected only mildly by the $C P$ phase such that the top quark-Higgs boson couplings with different $C P$ phases yield almost the same cut efficiencies. In the work, to demonstrate the judgement, we utilize the cuts in [8] named SR6 as a typical example which are listed as follows:

$$
\begin{aligned}
p_{T}^{l} & >20 \mathrm{GeV}, \quad p_{T}^{\text {jets }}>40 \mathrm{GeV}, \quad p_{T}^{b}>25 \mathrm{GeV} \\
\left|\eta^{e}\right| & <2.5, \quad\left|\eta^{\mu / \text { jets } / b}\right|<2.4 \\
N_{l} & =2, \quad N_{b} \geq 4, \quad N_{\text {jets }} \geq 5, \\
H_{T} & \geq 300 \mathrm{GeV}, \quad p_{T}^{\text {miss }}>50 \mathrm{GeV},
\end{aligned}
$$

where $H_{T}$ is scalar sum of all jets transverse momentum $p_{T}$ and $p_{T}^{\text {miss }}$ is the missing transverse momentum. Figure 2 displays efficiencies of the $t \bar{t} t \bar{t}$ production after applying all above cuts as a function of the $C P$ phase $\theta$ of $y_{t}$, where

$$
\theta \equiv \arctan \left(b_{t} / a_{t}\right)
$$

The horizontal dashed curve denotes the cut efficiency of gauge boson mediation contribution. While, the blue and red curve represents the cut efficiency of the interference contribution and the Higgs-boson mediation contribution, respectively. The three curves are close together in the

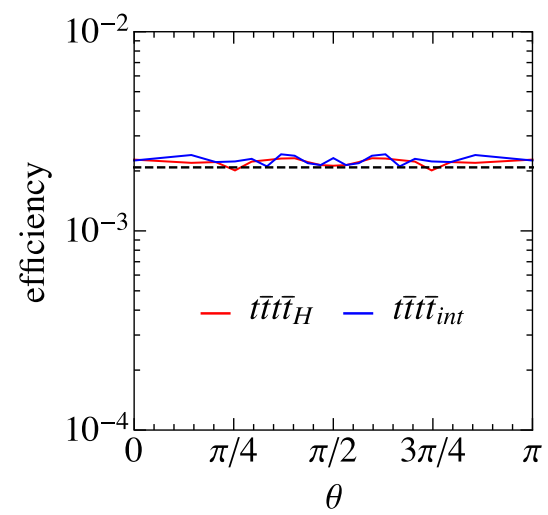

FIG. 2. The cut efficiency of the interference contribution (blue) and the Higgs-mediation contribution alone (red) in the $\bar{t} t \bar{t}$ production as a function of the $C P$ phase $\theta$. whole range of $C P$ phase $\theta$. Therefore, we can use the same strategy from the experimental collaboration to probe the potential of the LHC on the $y_{t}$. In our study, we adopt the cut flow used by the CMS Collaboration [8], which is optimized for searching the SM $t \bar{t} t \bar{t}$ production; see Eq. (6). The CMS study shows that there are 0.7 events of the SM background after all the cuts at the $13 \mathrm{TeV}$ LHC with an integrated luminosity $(\mathcal{L})$ of $35.9 \mathrm{fb}^{-1}$ [8]. We thus estimate the number of the SM background events $\left(N_{b}\right)$ as $N_{b}=0.0195 \mathcal{L}$ at the $13 \mathrm{TeV}$ LHC.

Next, we simulate the signal process of the four topquark production from the QCD, electroweak, and Higgs Yukawa interactions; see Eq. 2. We generate four top-quark events at the parton level using MadEvent [13] at the $13 \mathrm{TeV}$ LHC, and then pass them to Pythia [16] and Delphes [17] for parton showering, hadronization, and detector simulation. After that we apply cuts listed in Eq. (6) to the events and then obtain the cut efficiency. The signal events number is $N_{s}=\sigma(t \bar{t} t \bar{t}) \times \epsilon \times \mathcal{L}$ at a given integrated luminosity, where $\epsilon$ is the cut efficiency and $\mathcal{L}$ is the integrated luminosity. It is shown that 3.06 events of the signal $\left(N_{s}\right)$ in the $C P$-odd I $\left(a_{t}=0\right.$ and $\left.b_{t}=1\right)$ and 1.56 events of the background $\left(N_{b}\right)$ survive all the cuts with the current integrated luminosity of $80 \mathrm{fb}^{-1}$ in terms of $N_{b}$ and $N_{s}$ depicted above. As there are few events of both the signal and the backgrounds after the kinematics cuts, we estimate the significance of excluding the purely $C P$-odd $y_{t}$ coupling using [18]

$$
\sqrt{-2\left[N_{b} \log \frac{N_{s}+N_{b}}{N_{b}}-N_{s}\right]}=2 .
$$

Figure 3 displays the production cross section of the signal process as a function of the $y_{t}$ coupling, where the red and black curves denote the purely $C P$-odd coupling $b_{t}$ and

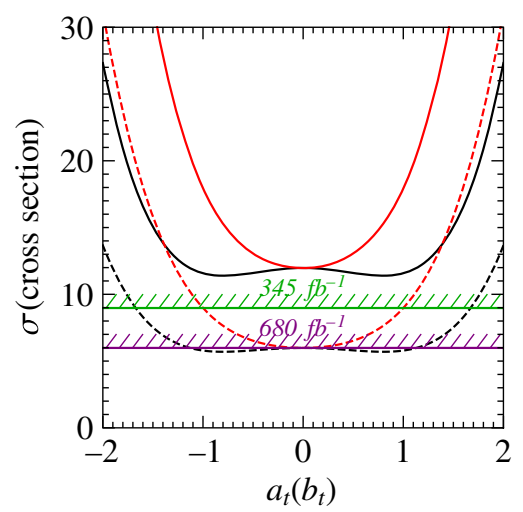

FIG. 3. Cross section of the $t \bar{t} t \bar{t}$ production as a function of a purely $C P$-even coupling $a_{t}$ (black) or a purely $C P$-odd coupling $b_{t}$ (red). The solid curves represent $\sigma_{0}$ while the dashed curves denote $\sigma_{0}-\delta \sigma_{\mu}$. The horizontal lines represent the upper limit of the production rate with an integrated luminosity of $345 \mathrm{fb}^{-1}$ (green) and $680 \mathrm{fb}^{-1}$ (purple). 

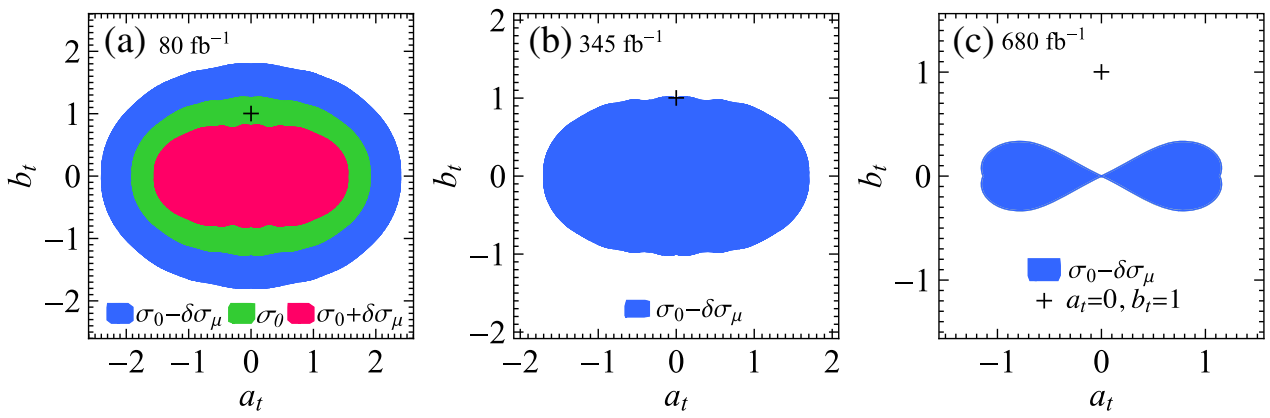

FIG. 4. Allowed parameter space in the plane of $a_{t}$ and $b_{t}$ at the $95 \%$ confidence level with an integrated luminosity of $80 \mathrm{fb}^{-1}$ (a), $345 \mathrm{fb}^{-1}$ (b), and $680 \mathrm{fb}^{-1}$ (c) if a null result of the $t \bar{t} t \bar{t}$ production is reported at the $13 \mathrm{TeV}$ LHC. The symbol " + " denotes the $C P$-odd I $\left(a_{t}=0\right.$ and $\left.b_{t}=1\right)$.

the purely $C P$-even coupling $a_{t}$, respectively. As mentioned above, the $t \bar{t} t \bar{t}$ production suffers from large scale dependences. For illustration, we plot both the central value of the signal production rate $\sigma_{0}$ (solid curve) and the lower value $\sigma_{0}-\delta \sigma_{\mu}$ (dashed curve). Those horizontal lines show the projected upper limit of the cross section of the $t \bar{t} t \bar{t}$ production obtained at the $13 \mathrm{TeV}$ LHC with an integrated luminosity of $345 \mathrm{fb}^{-1}$ (green) and $680 \mathrm{fb}^{-1}$ if only a null result is found in the four top-quark production measurement.

Figure 4 displays the allowed parameter space in the plane of $a_{t}$ and $b_{t}$ at the $95 \%$ confidence level for various integrated luminosities: (a) $80 \mathrm{fb}^{-1}$, (b) $345 \mathrm{fb}^{-1}$, and (c) $680 \mathrm{fb}^{-1}$. The cross symbol denotes the $C P$-odd I $\left(a_{t}=0\right.$ and $\left.b_{t}=1\right)$. The shaded regions are still allowed if the $t \bar{t} t \bar{t}$ is not observed for a given integrated luminosity, where the blue (green, red) region is obtained when considering the lower, central, and upper value of the signal production rate, respectively. Even though the current data cannot probe the $C P$-odd I when taking the scale uncertainty of the signal production into account, as shown in Fig. 4(a), collecting more data is able to constrain the $C P$-odd coupling. For example, one can exclude the $C P$-odd I at the $2 \sigma$ C.L., even considering the lower value of the signal production, when an integrated luminosity of $345 \mathrm{fb}^{-1}$ is accumulated; see Fig. 4(b). Furthermore, $C P$-odd II can be excluded when the integrated luminosity reaches $680 \mathrm{fb}^{-1}$, which is depicted in Fig. 4(c).

\section{GLOBAL ANALYSIS OF MULTITOP PRODUCTIONS}

Now we turn to the case of $C P$-mixture coupling. The $C P$ phase of $y_{t}$ plays different roles in various Higgs-boson production channels, e.g., the single-Higgs production, the $t \bar{t} H$ associated production, and the $t \bar{t} t \bar{t}$ production. For example, the $t \bar{t} H$ production is modified as [19]

$$
\frac{\sigma(g g \rightarrow t \bar{t} H)}{\sigma(g g \rightarrow t \bar{t} H)_{\mathrm{SM}}} \equiv \mu_{t \bar{t} H}^{\mathrm{Th}}=a_{t}^{2}+0.46 b_{t}^{2},
$$

while the single Higgs-boson production through gluon fusion $(g g \rightarrow H)$ is modified as [19]

$$
\frac{\sigma(g g \rightarrow H)}{\sigma(g g \rightarrow H)_{\mathrm{SM}}} \equiv \mu_{g g \rightarrow H}^{\mathrm{Th}}=a_{t}^{2}+2.29 b_{t}^{2} .
$$

Obviously, the scalar component of the $y_{t}$ coupling dominates in the former process while the pseudoscalar dominates in the latter. Therefore, combining the two Higgs-boson production channels with the $t \bar{t} t \bar{t}$ production would yield much tighter constraints on $y_{t}$.

When dealing with the signal strength measured in various Higgs-boson production channels, one needs to pay some attention to the Higgs-boson width effect, which could dramatically alter the branching ratios of the Higgs-boson decay mode. Define $R_{\Gamma}$ as the ratio of the Higgs-boson width $\left(\Gamma_{H}\right)$ to the SM value $\left(\Gamma_{H}^{\mathrm{SM}}\right)$, i.e.,

$$
R_{\Gamma} \equiv \Gamma_{H} / \Gamma_{H}^{\mathrm{SM}} .
$$

The ratio describes the new physics effects either from the modification of the Higgs-boson couplings to the SM particles, or from unknown decay modes such as the invisible Higgs-boson decay in the Higgs-portal dark matter models $[20,21]$. Recently, the $t \bar{t} H$ production is measured in final states with electrons, muons, and hadronically decaying $\tau$ leptons by the CMS Collaboration [22]. As those leptonic modes are not sensitive to the top quark-Higgs boson couplings, we can parametrize the signal strength in the $t \bar{t} H$ production as

$$
\mu_{\bar{t} \bar{H}}=\left(a_{t}^{2}+0.46 b_{t}^{2}\right) / R_{\Gamma}
$$

On the other hand, the best mode to measure the singleHiggs production is through the diphoton mode $H \rightarrow \gamma \gamma$, which is also affected by $y_{t}$. Using the so-called narrow width approximation, we parametrize the signal strength of $g g \rightarrow H \rightarrow \gamma \gamma$ as 


$$
\begin{aligned}
& \frac{\sigma(g g \rightarrow H \rightarrow \gamma \gamma)}{\sigma(g g \rightarrow H \rightarrow \gamma \gamma)_{\mathrm{SM}}} \\
& =\left(a_{t}^{2}+2.29 b_{t}^{2}\right) \times \frac{0.0237\left(-8.33+1.83 a_{t}\right)^{2}+0.185 b_{t}^{2}}{R_{\Gamma}} .
\end{aligned}
$$

It is shown that a global analysis of combining multitop productions can probe $\Gamma_{H}$ from the above analysis.

The recent measurement of the $t \bar{t} H$ production and the $g g \rightarrow H \rightarrow \gamma \gamma$ process by the CMS Collaborations yields $[3,22]$

$$
\begin{aligned}
\mu_{t \bar{t} H}^{\operatorname{Exp}} & =0.96_{-0.31}^{+0.34}, & & \mathcal{L}=41.5 \mathrm{fb}^{-1}, \\
\mu_{g g \rightarrow H \rightarrow \gamma \gamma}^{\operatorname{Exp}} & =1.16_{-0.18}^{+0.21}, & & \mathcal{L}=36.6 \mathrm{fb}^{-1} .
\end{aligned}
$$

As very complicated statistical methods have been used in the experimental analysis, it is difficult for us to estimate how large the statistical uncertainties should be when collecting more data. Therefore, we adopt the uncertainties shown above to explore the sensitivities of the LHC with an integrated luminosity of $300 \mathrm{fb}^{-1}$.

We begin with the case of $R_{\Gamma}=1$, i.e., $\Gamma_{H}=\Gamma_{H}^{\mathrm{SM}}$. In Fig. 5(a), we plot the parameter space in the plane of $a_{t}$ and $b_{t}$ that is consistent with the current measurements of the $g g \rightarrow H \rightarrow \gamma \gamma$ channel (green) and the $t \bar{t} H$ production (blue) at the $1 \sigma$ confidence level. The blue ellipse of the $t \bar{t} H$ production centers around $\left(a_{t}, b_{t}\right)=(0,0)$ as expected from Eq. (12). On the other hand, the $g g \rightarrow H \rightarrow \gamma \gamma$ channel is affected by the $y_{t}$ coupling in both the production and decay. That makes the green oval shifts towards to positive $a_{t}$ region; see Eq. (13). For the four top-quark $(t \bar{t} t \bar{t})$ production collider simulation, we apply the same procedure as the purely $C P$-odd case. The red region denotes the allowed parameter space at the 95\% C.L. if only null results were reported in the search of the $t \bar{t} t \bar{t}$ event at the $13 \mathrm{TeV}$ LHC with an integrated luminosity of $300 \mathrm{fb}^{-1}$. Note that the $t \bar{t} t \bar{t}$ production suffers from a large scale dependence in the theoretical prediction of the production cross section. In order to make a conservative estimation of the parameter space, we use the lower value of $\sigma(t \bar{t} t \bar{t})$ allowed by the scale dependence, i.e., $\sigma_{0}-\delta \sigma_{\mu}$, in our global analysis. It turns out that the bound from the $t \bar{t} t \bar{t}$ production is loose in comparison with the constraints from the other two channels. As a result, the allowed parameter space is determined by the $g g \rightarrow H$ and the $t \bar{t} H$ productions. The bounds on $a_{t}$ and $b_{t}$ read as

$$
0.73<a_{t}<1.14, \quad-0.51<b_{t}<0.51 .
$$

That corresponds to a constraint on the $C P$ phase of $y_{t}$ as following:

$$
-\frac{\pi}{5} \leq \theta \leq \frac{\pi}{5}
$$
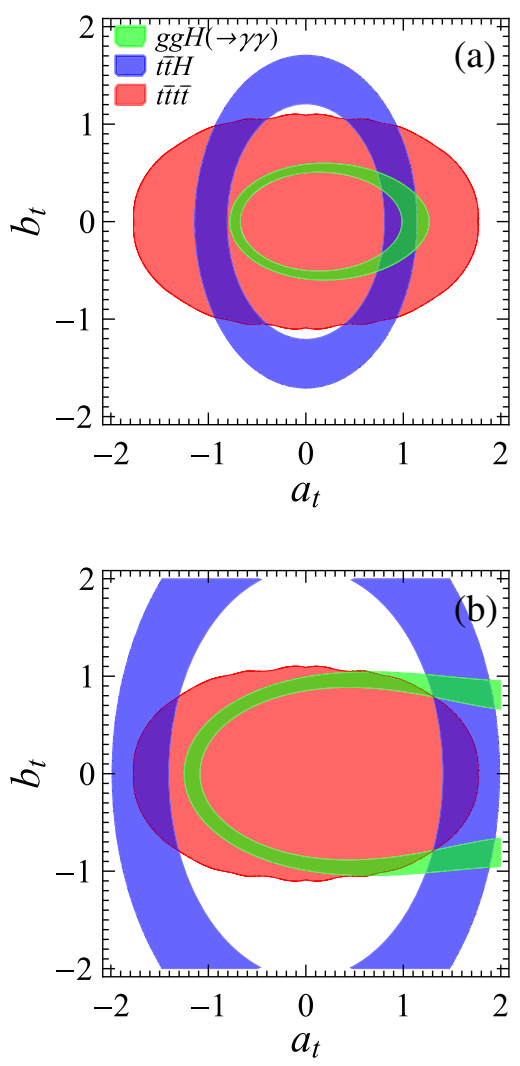

FIG. 5. Parameter space in the plane of $a_{t}$ and $b_{t}$ consistent with the current measurements of $g g H(\rightarrow \gamma \gamma)$ (green) and the $\bar{t} H$ production (blue), and the projection of the $t \bar{t} t \bar{t}$ production (red) if a null result is reported at the $\mathrm{LHC}$ with $\mathcal{L}=300 \mathrm{fb}^{-1}$. We fix $R_{\Gamma}=1$ in the Fig. (a) but relax it in the Fig. (b).

Making use of the fact that the shape of parameter space of the diphoton channel is sensitive to the $y_{t}$ coupling, we can vary the $R_{\Gamma}$ parameter to check the consistence of the three channels. As shown in Eq. (13), increasing $\Gamma_{H}$ requires larger values of $a_{t}$ and $b_{t}$ to compensate the suppression effect of $\Gamma_{H}$ in the denominator. Meanwhile, the cancellation between the $W$-boson loop and the topquark loop further distorts the shape of the green eclipse. For a large $R_{\Gamma}$, one cannot even find a common parameter space consistent with the three channels; see Fig. 5(b). That yields a constraint on the Higgs-boson width as

$$
R_{\Gamma} \leq 3.04
$$

or

$$
\Gamma_{H} \leq 3.04 \times \Gamma_{H}^{\mathrm{SM}} \simeq 12.19 \mathrm{MeV},
$$

which is comparable to the upper limits of the Higgs total width given by the ATLAS and the CMS Collaborations $[23,24]$ which determine the Higgs-boson width from the comparison of the on shell and off shell effects in the $g g \rightarrow H \rightarrow Z Z^{*}$ channel.

Finally, we explore the potential of the high luminosity phase of the LHC (HL-LHC) which is going to collect an 

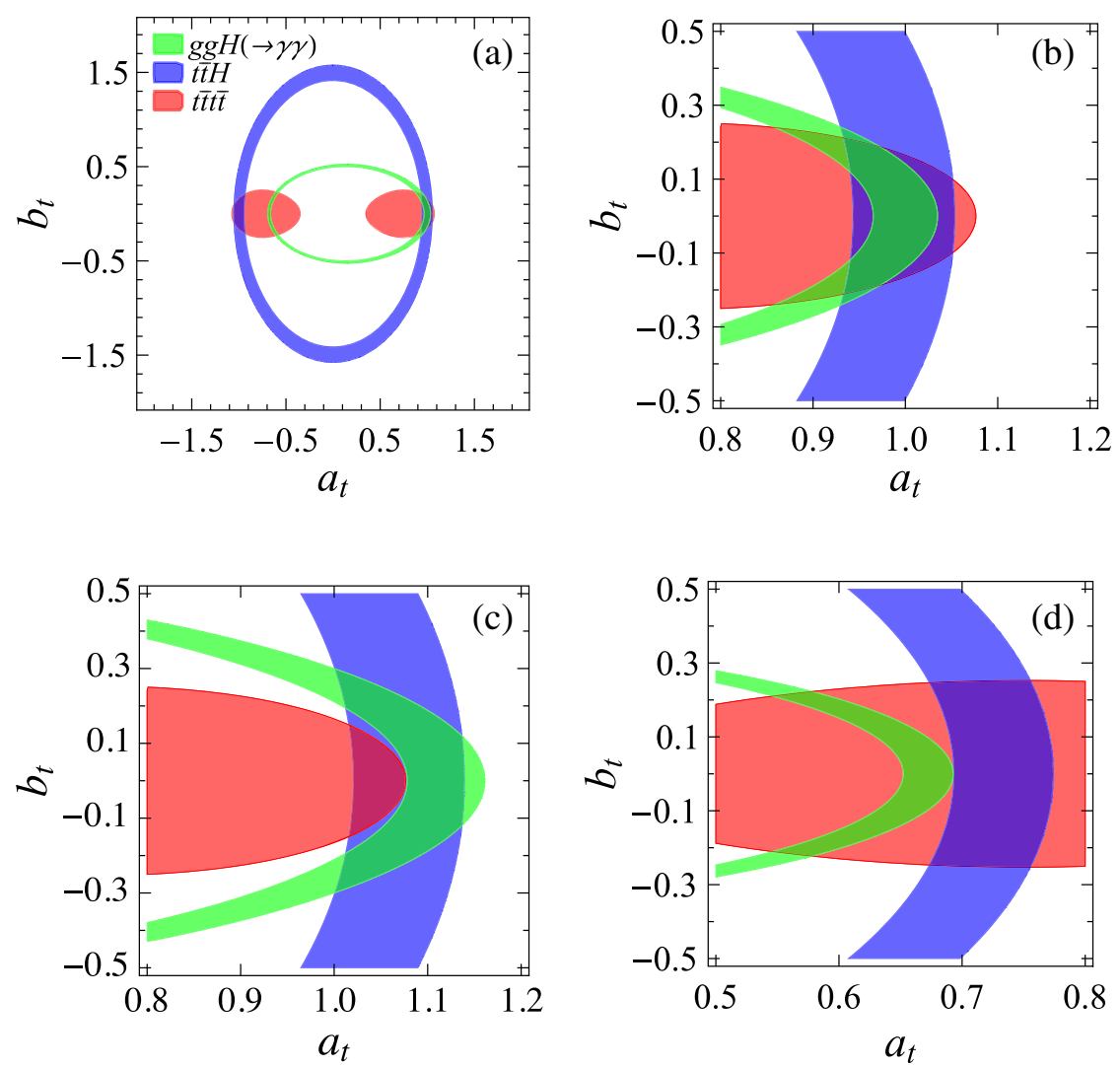

FIG. 6. Parameter space consistent with the measurement of $g g H(\rightarrow \gamma \gamma)$ (green) and $t \bar{t} H$ (blue) productions at the $1 \sigma$ C.L., while the red region is allowed by the $t \bar{t} t \bar{t}$ production measurement with $50 \%$ uncertainty at the $13 \mathrm{TeV}$ LHC with an integrated luminosity of $3000 \mathrm{fb}^{-1}$. In (a) and (b), the Higgs boson total width is taken to be the same as the SM prediction, while in (c) 1.17 times and (d) 0.54 times as the SM prediction.

integrated luminosity of $3000 \mathrm{fb}^{-1}$. Thanks to the unprecedented high luminosity, the $g g \rightarrow H \rightarrow \gamma \gamma$ and $t \bar{t} H$ productions can be measured very precisely. For example, it is shown that [25]

$$
\begin{aligned}
\mu_{t \bar{t} H}^{\mathrm{HL}} & =1.00_{-0.11}^{+0.11}, \\
\mu_{g g \rightarrow H \rightarrow \gamma \gamma}^{\mathrm{HL}} & =1.00_{-0.05}^{+0.05} .
\end{aligned}
$$

In addition, the $t \bar{t} t \bar{t}$ production can also be discovered, and we assume the contribution from the Higgs-boson mediation can be measured with an accuracy of $50 \%$. Figure 6 displays the parameter space in the plane of $a_{t}$ and $b_{t}$ allowed by the projected measurements of the $g g \rightarrow H \rightarrow$ $\gamma \gamma$ process (green), the $t \bar{t} H$ production (blue), and the $t \bar{t} t \bar{t}$ production (red), assuming $R_{\Gamma}=1$. Now the $t \bar{t} t \bar{t}$ channel yields two isolated ovals in the plane of $\left(a_{t}, b_{t}\right)$, of which only the one around the SM value $\left(a_{t}=1\right.$ and $\left.b_{t}=0\right)$ is allowed. See Fig. 6(b) for the details. Combing the three channels yields a constraint on $a_{t}$ and $b_{t}$ as follows:

$$
0.93<a_{t}<1.08, \quad-0.21<b_{t}<0.21,
$$

which corresponds to

$$
-\frac{2 \pi}{27} \lesssim \theta \lesssim \frac{2 \pi}{27}
$$

Again, setting $R_{\Gamma}$ as a free parameter sizably alters the parameter space of the diphoton channel and gives rise to a tight constraint on the Higgs-boson width. For $R_{\Gamma}>1$, the green eclipse stretches toward to a large $a_{t}$ region; see Fig. 6(c). A good news is that the HL-LHC is able to probe a small $R_{\Gamma}$. If the Higgs-boson width is smaller than the SM value, i.e., $R_{\Gamma}<1$, a smaller value of $a_{t}$ and $b_{t}$ are needed, and for that reason, the green oval shrinks; see Fig. 6(d). By varying $R_{\Gamma}$, we are able to obtain a more stringent bound on $R_{\Gamma}$ as following:

$$
0.54 \leq R_{\Gamma} \leq 1.17
$$

i.e.,

$$
2.2 \mathrm{MeV} \lesssim \Gamma_{H} \lesssim 4.7 \mathrm{MeV} .
$$

\section{SUMMARY}

The multitop production is a powerful tool to probe the $C P$ property of the top quark-Higgs boson interaction, 


$$
\mathcal{L}_{t \bar{t} H}=-\frac{m_{t}}{v} H \bar{t}\left(a_{t}+i b_{t} \gamma_{5}\right) t
$$

and the Higgs-boson width $\Gamma_{H}$ at the LHC. After considering the large scale uncertainty $(\sim 50 \%)$ of the signal rate, we showed that the four top-quark production alone can exclude $C P$-odd I top-quark Yukawa coupling $\left(a_{t}=0\right.$ and $\left.b_{t}=1\right)$ at the $13 \mathrm{TeV}$ LHC with an integrated luminosity of $345 \mathrm{fb}^{-1}$. Furthermore, increasing the integrated luminosity to $680 \mathrm{fb}^{-1}$ excludes a purely $C P$-odd coupling, regardless the size of the Yukawa coupling.

As the top-quark Yukawa coupling and the Higgs-boson width play different roles in various Higgs-boson productions, combining different channels would impose tighter constraints on the $C P$ phase $\theta \equiv \arctan \left(b_{t} / a_{t}\right)$ and Higgsboson width. In this study, we considered three production channels as follows:

(1) the single-Higgs production with a subsequent decay of $H \rightarrow \gamma \gamma$,

(2) the $t \bar{t} H$ associated production,

(3) the four-top production.

After considering the current and projected experimental measurements, we showed that, assuming the Higgs-boson width is not altered by the new physics effect, the $C P$ phase is bounded to be $|\theta| \leq \frac{\pi}{5}$, if only a null result is found in the search of $t \bar{t} t \bar{t}$ events at the LHC with an integrated luminosity of $300 \mathrm{fb}^{-1}$. Furthermore, varying the Higgsboson width dramatically distorts the parameter space of the $g g \rightarrow H \rightarrow \gamma \gamma$ channel and even leads to nonconsistent parameter space for all the three channels. We found that $\Gamma_{H} \leq 3.04 \Gamma_{H}^{\mathrm{SM}} \simeq 12.19 \mathrm{MeV}$.

At the high luminosity phase of the LHC with an integrated luminosity of $3000 \mathrm{fb}^{-1}$, all the three channels are expected to be measured precisely. We demonstrated that, if the Higgs-boson related $t \bar{t} t \bar{t}$ production is measured with an uncertainty of $50 \%$, the combined analysis yields stringent constraints on the $C P$ phase as $|\theta| \lesssim 2 \pi / 27$ with the assumption of $\Gamma_{H}=\Gamma_{H}^{\mathrm{SM}}$. Varying the Higgs-boson width yields $2.2 \mathrm{MeV} \lesssim \Gamma_{H} \lesssim 4.7 \mathrm{MeV}$.

\section{ACKNOWLEDGMENTS}

The work is supported in part by the National Science Foundation of China under Grants No. 11175069, No. 11275009, No. 11422545, No. 11725520, No. 11675002, No. 1163500, No. 11775093, No. 11805013, and the Fundamental Research Funds for the Central Universities under Grant No. 2018NTST09.
[1] M. Aaboud et al. (ATLAS Collaboration), Phys. Lett. B 784, 173 (2018).

[2] A. M. Sirunyan et al. (CMS Collaboration), Phys. Rev. Lett. 120, 231801 (2018).

[3] A. M. Sirunyan et al. (CMS Collaboration), Eur. Phys. J. C 79, 421 (2019).

[4] A. Kobakhidze, L. Wu, and J. Yue, J. High Energy Phys. 10 (2014) 100.

[5] M. R. Buckley and D. Goncalves, Phys. Rev. Lett. 116, 091801 (2016).

[6] D. Gonçalves, K. Kong, and J. H. Kim, J. High Energy Phys. 06 (2018) 079.

[7] Q.-H. Cao, S.-L. Chen, and Y. Liu, Phys. Rev. D 95, 053004 (2017).

[8] A. M. Sirunyan et al. (CMS Collaboration), Eur. Phys. J. C 78, 140 (2018).

[9] R. Frederix, D. Pagani, and M. Zaro, J. High Energy Phys. 02 (2018) 031.

[10] R. Contino et al., CERN Report No. CERN-TH-2016-113, 2017, pp. 255-440.

[11] F. Caola and K. Melnikov, Phys. Rev. D 88, 054024 (2013).

[12] N. P. Hartland, F. Maltoni, E. R. Nocera, J. Rojo, E. Slade, E. Vryonidou, and C. Zhang, J. High Energy Phys. 04 (2019) 100.

[13] J. Alwall, P. Demin, S. de Visscher, R. Frederix, M. Herquet, F. Maltoni, T. Plehn, D. L. Rainwater, and T. Stelzer, J. High Energy Phys. 09 (2007) 028.
[14] G. Bevilacqua and M. Worek, J. High Energy Phys. 07 (2012) 111.

[15] J. Alwall, R. Frederix, S. Frixione, V. Hirschi, F. Maltoni, O. Mattelaer, H. S. Shao, T. Stelzer, P. Torrielli, and M. Zaro, J. High Energy Phys. 07 (2014) 079.

[16] T. Sjöstrand, S. Ask, J. R. Christiansen, R. Corke, N. Desai, P. Ilten, S. Mrenna, S. Prestel, C. O. Rasmussen, and P.Z. Skands, Comput. Phys. Commun. 191, 159 (2015).

[17] J. de Favereau, C. Delaere, P. Demin, A. Giammanco, V. Lematre, A. Mertens, and M. Selvaggi (DELPHES 3 Collaboration), J. High Energy Phys. 02 (2014) 057.

[18] G. Cowan, K. Cranmer, E. Gross, and O. Vitells, Eur. Phys. J. C 71, 1 (2011); 73, 2501(E) (2013).

[19] F. Boudjema, R. M. Godbole, D. Guadagnoli, and K. A. Mohan, Phys. Rev. D 92, 015019 (2015).

[20] B. Patt and F. Wilczek, arXiv:hep-ph/0605188.

[21] C. Q. Geng and J. N. Ng, Mod. Phys. Lett. A 04, 581 (1989).

[22] T. C. Collaboration (CMS Collaboration), CERN Report No. CMS-PAS-HIG-18-019, 2018.

[23] M. Aaboud et al. (ATLAS Collaboration), Phys. Lett. B 786, 223 (2018).

[24] A. M. Sirunyan et al. (CMS Collaboration), arXiv:1901 .00174 .

[25] R. Venditti (CMS Collaboration), EPJ Web Conf. 192, 00032 (2018). 